\title{
Síntese e caracterização de hidroxiapatita natural extraída de escamas de pirarucu (Arapaima gigas)
}

\section{Synthesis and characterization of natural hydroxyapatite extracted from pirarucu scales (Arapaima gigas)}

\author{
Mateus Oliveira de Amorim ${ }^{1}$, Karyane Meazza ${ }^{2}$, \\ Ana Alice Oliveira ${ }^{3}$, José Carlos Calado Sales Júnior ${ }^{4}$, \\ Yurimiler Leyet Ruiz ${ }^{5}$, Jean Carlos Silva Andrade ${ }^{6}$
}

\footnotetext{
${ }^{1}$ Departamento de Engenharia de Materiais - DEMA/UFAM, Campus Universitário Senador Arthur Virgílio Filho - Av. General Rodrigo Octávio, 3000 - Coroado I, CEP: 69.077-000, Manaus, AM, Brasil. e-mail: mateusoliveiradeamorim@gmail.com

${ }^{2}$ Programa de Pós-Graduação em Ciência e Engenharia de Materiais - PPGCEM/UFAM, Campus Universitário Senador Arthur Virgílio Filho, Av. General Rodrigo Octávio, 6200 - Coroado I - CEP: 69.077-000, Manaus, AM, Brasil. e-mail: karyanemeazza@gmail.com

${ }^{3}$ Departamento de Engenharia de Materiais - DEMA/UFAM, Campus Universitário Senador Arthur Virgílio Filho - Av. General Rodrigo Octávio, 3000 - Coroado I, CEP: 69.077-000, Manaus, AM, Brasil.

e-mail: barros.nalice@gmail.com

${ }^{4}$ Departamento de Engenharia de Materiais - DEMA/UFAM, Campus Universitário Senador Arthur Virgílio Filho - Av. General Rodrigo Octávio, 3000 - Coroado I, CEP: 69.077-000, Manaus, AM, Brasil.

e-mail: jccalado@gmail.com

${ }^{5}$ Programa de Pós-Graduação em Ciência e Engenharia de Materiais - PPGCEM, DEMA/UFAM, Campus Universitário Senador Arthur Virgílio Filho, Av. General Rodrigo Octávio, 6200 - Coroado I - CEP: 69.077-000, Manaus, AM, Brasil. e-mail: yurileyet@gmail.com

${ }^{6}$ Programa de Pós-Graduação em Ciência e Engenharia de Materiais - PPGCEM, DEMA/UFAM, Campus Universitário Senador Arthur Virgílio Filho, Av. General Rodrigo Octávio, 6200 - Coroado I - CEP: 69.077-000, Manaus, AM, Brasil. e-mail: jean.engmateriais@gmail.com
}

\section{RESUMO}

A hidroxiapatita integra o grupo de biomateriais cerâmicos sendo aplicada na área médica devido a sua biocompatibilidade e propriedade osteocondutora, o que a torna uma opção para tratamentos envolvendo recuperação de traumas ósseos. Os resíduos pesqueiros, os ossos e as escamas, podem ser utilizados como uma fonte barata para a produção de fosfatos de cálcio (hidroxiapatita). O objetivo do estudo foi o uso das escamas de pirarucu, resíduo não tóxico, para investigar a viabilidade de extrair hidroxiapatita natural e caracterizá-la por análises físico-químicas e estruturais a fim de comprovar a sua integridade. Primeiramente, foi realizado caracterização térmica para estabelecer as condições do tratamento térmico. Com isso, as escamas foram tratadas termicamente para a remoção dos compostos orgânicos. Após isso, o material foi homogeneizado, peneirado e purificado com reagentes, devido a possibilidade da amostra absorver impurezas durante o procedimento experimental. As caracterizações físico-químicas e estruturais foram realizadas para acompanhar e comprovar as etapas desenvolvidas e examinadas. Os resultados apresentam que o produto obtido apresentou 99\% de fase de HAp natural e fase cristalina. Na região do infravermelho obteve-se a presença dos picos $3750-3500 \mathrm{~cm}^{-1}$ e $610-560 \mathrm{~cm}^{-1}$, referentes aos íons $\mathrm{OH}^{-}$e $\mathrm{PO}_{4}^{-3}$ respectivamente, apresentando os grupos funcionais hidroxila e fosfato que constituem a fórmula química da hidroxiapatita e, por meio da espectroscopia por energia dispersiva, as amostras apresentaram percentuais elementares de cálcio (37\%), oxigênio (40\%) e fósforo (13) que são compatíveis com a razão molar $\mathrm{Ca} / \mathrm{P}$ da fase de hidroxiapatita.

Palavras-chave: escamas, tratamento térmico, hidroxiapatita natural, grupos funcionais, fase cristalina.

\section{ABSTRACT}

The hydroxyapatite belongs to the group of ceramic biomaterials being applied in the medical area due to its 
biocompatibility and osteoconductive properties, which makes it an option for treatments involving recovery of bone traumas. Fishing residues, bones and scales can be used as an inexpensive source for the production of calcium phosphates (hydroxyapatite). The objective of the study was the use of the non-toxic residue pirarucu scales to investigate the viability of extracting natural hydroxyapatite and characterizing it by physicochemical and structural analyzes in order to prove its integrity. Firstly, thermal characterization was performed to establish the conditions of the heat treatment. With this, the scales were heat treated for the removal of the organic compounds. Thereafter, the material was homogenized, sieved and purified with reagents, because the sample could absorb impurities during the experimental procedure. The physical-chemical and structural characterizations were performed to accompany and prove the stages developed and examined. The results showed that the product obtained showed $99 \%$ of natural HAp phase and crystalline powders. In the infrared region, the peaks $3750-3500 \mathrm{~cm}^{-1}$ and $610-560 \mathrm{~cm}^{-1}$ were obtained referring to the $\mathrm{OH}-$ and PO4-3 ions respectively, referring to the functional groups hydroxyl and phosphate that constitute the chemical formula of hydroxyapatite and, by means of dispersive energy spectroscopy, the samples presented elemental percentages of calcium (37\%), oxygenus (40\%) and phosphorus (13\%) that are compatible with the $\mathrm{Ca} / \mathrm{P}$ molar ratio of the hydroxyapatite phase.

Keywords: scales, thermal treatment, natural hydroxyapatite, functional groups, crystalline phase.

\section{INTRODUÇÃO}

Os biomateriais cerâmicos são classificados de acordo com a sua interação com o tecido hospedeiro, sendo divididos em bioinertes, bioativos e bioabsorvíveis. Os bioinertes, com base em suas propriedades físicas e mecânicas, são tolerados pelo organismo após serem implantados, sem resposta do organismo. Os bioabsorvíveis são substituídos por células em crescimento devido ao processo de degradação relacionado ao metabolismo. Os bioativos auxiliam na cura devido a interagirem com o tecido circundante o que resulta no organismo em responder ao implante como se fosse um tecido natural [1]. Nessa última divisão que enquadra-se a hidroxiapatita $\left[\mathrm{Ca}_{10}\left(\mathrm{PO}_{4}\right)_{6}(\mathrm{OH})_{2}\right]$, se tratando de um fosfato de cálcio que, quando aplicado em implantes, proporciona um microambiente condutivo, contribuindo a formação e crescimento ósseo [1]. Além disso, as células do organismo não são capazes de distinguir a hidroxiapatita da superfície óssea, indicando a ocorrência de similaridade química superficial entre ambos.

A limitação da hidroxiapatita está relacionada à sua lenta biodegradação, que ocorre por mecanismos celulares gradualmente após 4-5 anos após realizado o implante. Este comportamento pode ser atribuído à razão molar $\mathrm{Ca} / \mathrm{P}$ de 1,67, que torna a HAp praticamente insolúvel em meios neutros [1].

A literatura abrange pesquisas que exploram escamas de peixes como matéria-prima que podem ser empregadas para a obtenção de biomateriais cerâmicos. A indústria do pescado, segundo dados da FAO (2016) [2], tem apontado perspectivas promissoras devido as demandas existentes nos mercados da Ásia, África, América Latina e Caribe, isso contrasta com as dificuldades que o setor pesqueiro enfrenta, principalmente no que diz respeito aos resíduos provenientes do processo do pescado, com destaque para as escamas que são resíduos sólidos que podem corresponder a 1\% da massa total do peixe [3].

A similaridade química e estrutural da hidroxiapatita com componentes minerais presentes nos ossos de humanos e nos dentes a torna um biomaterial reconhecido e amplamente aplicado em implantes [4], devido as seguintes propriedades de osteocondutividade, osteogênese, osteointegração e biocompatibilidade [5]. Os elementos que constituem a HAp são principalmente cálcio, fósforo e oxigênio, sendo a razão molar de cálcio por fósforo de 1,67. Apresenta a fórmula molecular de $\left[\mathrm{Ca}_{10}\left(\mathrm{PO}_{4}\right)_{6}(\mathrm{OH})_{2}\right]$, sendo um composto formado por uma célula unitária hexagonal constituída por dez íons de cálcio em sítios não equivalentes, nos quais comportam quatro no sítio I (tetraédrico - Ca I) e seis no sítio II (octaédricos - Ca II). O sítio I é composto pelos íons de cálcio alinhados em colunas, já no sítio II os íons estão em triângulos equiláteros perpendiculares à direção do plano $\mathrm{C}$ da estrutura. Além disso, a estrutura é compreendida por tetraedros de $\mathrm{PO}_{4}{ }_{4}^{3-}$, no qual os íons de cálcio do sítio I são coordenados a seis átomos de oxigênio pertencentes a diferentes tetraedros do íon fosfato [6]. Com isso, a célula unitária apresenta seis grupos fosfato $-\mathrm{PO}_{4}$ e dois grupos hidroxila - OH, neste caso, os sítios cristalinos de oxigênio são designados como $\mathrm{O} 1, \mathrm{O} 2, \mathrm{O} 3$ e O4, sendo o $\mathrm{O} 4$ o oxigênio do grupo da hidroxila, já os demais são ligados ao fósforo - P. Os cristais de HAp naturais possuem célula unitária com parâmetros de rede: $\mathrm{a}=\mathrm{b}=9,389 \AA \mathrm{e} \mathrm{c}=6,869 \AA, \alpha=\beta=90^{\circ} \mathrm{e} \gamma=120^{\circ}$ [6].

As formas de obtenção da hidroxiapatita são várias e para cada método aplicado resultará em propriedades distintas, com isso é necessário uma escolha adequada para que ocorra o controle da estrutura e que o produto final atenda ao propósito do estudo [7]. Diversos autores relatam a obtenção de hidroxiapatita por rotas de síntese por meio seco, via úmida, processos de baixas ou altas temperaturas, síntese com base em 
fontes biogênicas e processos combinados [4] [5] [8] [9], sendo tais métodos com uso de ácidos, álcalis e/ou calor para o maior rendimento da separação da hidroxiapatita de fontes biológicas [10].

A espécie Arapaima gigas é endêmica da Região Amazônica alcançando duzentos quilogramas de massa e três metros de comprimento [11]. São encontrados no Peru, Bolívia, Colômbia, Guiana e Brasil, em território nacional, preferencialmente nas bacias hidrográficas do Rio Amazonas e Tocantins-Araguaia, tem habitat em águas calmas de terras baixas, denominadas várzeas.

A produção do pescado de pirarucu, segundo dados IBGE (2015), gira em torno de oito milhões de quilogramas de quantidade produzida, com valor de produção em torno de oitotenta e cinco mil reais, considerando o potencial do pirarucu para a psicultura devido ao seu valor social, econômico e gastronômico na região amazônica [12] [13].

Os principais obstáculos que integram as dificuldades de consolidar o cuidado com a espécie é a intensa exploração nos estoques naturais e a falta de uma fiscalização da pesca e comércio eficaz em toda a região. A pesca predatória da espécie ocasionou no desaparecimento desses animais em ambientes que antes eram encontrados [14], devido a isso, surgiram regulamentações e estratégias de conservação somadas as regras de ordenamentos pesqueiros que são atitudes que estão sendo tomadas para evitar o desaparecimento da espécie [15] [16]. Outro ponto notável, a respeito do cultivo e reprodução, gira em torno de poucos estudos que informam a biologia reprodutiva da espécie, logo informações científicas contribuiriam para a elaboração de procedimentos com o objetivo de controlar a reprodução e consequentemente influenciaria na produção industrial do pirarucu.

Neste contexto, busca-se uma rota alternativa para a obtenção da hidroxiapatita natural a partir das escamas do Arapaima gigas, de modo a somar conhecimento a comunidade científica, sendo que há pesquisas sobre a estrutura e composição das escamas, mas escassas no diz respeito como matéria-prima para produção de biocerâmicas. Portanto, é essencial que este estudo apresente resultados coesos que sustentem a viabilidade de extrair hidroxiapatita natural das escamas do pirarucu.

\section{MATERIAIS E MÉTODOS}

\subsection{Matéria-prima}

As escamas foram coletadas na empresa Iranduba Frigorífico de Pescados LTDA. Em seguida foram lavadas com água corrente, submersas em água morna $\left(60^{\circ} \mathrm{C}\right)$ por 30 minutos e submetidas a limpeza manual com escova para remoção das partes grosseiras (rebarbas). Após isso, foram separadas e secas em estufa por 24 horas $/ 100^{\circ} \mathrm{C}$.

\subsection{Caracterização Térmica}

A caracterização térmica por termogravimetria foi realizada para estabelecer a temperatura desejada para a realização do tratamento térmico (calcinação).

O equipamento utilizado foi o modelo SDT Q600 v20.9 Build 20, com taxa de aquecimento de $10^{\circ} \mathrm{C} / \mathrm{min}$, atmosfera de $\mathrm{N}(30 \mathrm{~mL} / \mathrm{min})$ em intervalo de temperatura $25^{\circ} \mathrm{C}$ a $1000^{\circ} \mathrm{C}$ com amostra contendo $14 \mathrm{mg}$ da escama armazenada em um cadinho de alumina com volume total de $90 \mu \mathrm{L}$.

\subsection{Tratamento térmico e homogeneização}

Com base na caracterização térmica, foi estabelecida a temperatura de $700^{\circ} \mathrm{C}$ e taxa de aquecimento de $10^{\circ} \mathrm{C} /$ min para a realização da calcinação, com variação do tempo de permanência na mufla em 2,4 e 6 horas. O equipamento utilizado para o tratamento térmico foi o modelo Forno Mufla GP Científica com temperatura máxima de aquecimento de $1500^{\circ} \mathrm{C}$.

Após finalizado o tratamento térmico, o material foi removido da mufla, homogeneizado em almofariz de ágata e peneirado com granulometria passante de 200 mesh (abertura de $0,075 \mathrm{~mm}$ ). Nessa etapa, foram obtidas três amostras em pó: amostra com 2 horas, amostra com 4 horas e amostra com 6 horas de tratamento térmico.

\subsection{Processo de remoção de impurezas}

A próxima etapa compreendeu a pesagem de $20 \mathrm{~g}$ do pó das amostras tratadas termicamente. Cada amostra ( $2 \mathrm{~h}, 4 \mathrm{~h}$ e $6 \mathrm{~h}$ ) foi submetida a concentração de $0,1 \mathrm{M} / \mathrm{L}$ de ácido clorídrico por $1 \mathrm{~h} / 600 \mathrm{rpm}$ em temperatura ambiente. Em seguida, a solução foi neutralizada com água destilada e adicionou-se solução de $\mathrm{NaOH}(5 \%$ $\mathrm{m} / \mathrm{v}, 600 \mathrm{rpm}, 70^{\circ} \mathrm{C}$ ) por $5 \mathrm{hrs}$. Após esse procedimento, a solução passou pelo processo de esfriamento, para depois ser realizada a aplicação do ultrassom com $55 \%$ de potência com $0,2 \mathrm{~g} /$ e $100 \mathrm{ml}$ de etanol por trinta 
minutos para cada amostra e, após isso, realizaram-se os procedimentos de filtragem, secagem $\left(100^{\circ}\right.$ por 24 horas), homogeneização com almofariz de ágata e, por fim, peneiramento.

Esta etapa é uma cautela em se obter um produto final refinado e com mínimo de impurezas, devido a possibilidade de absorção de resíduos presentes nos equipamentos pela amostra ou a contaminação do material com os utensílios utilizados no experimento e preparação das amostras.

Por fim, as três amostras ( $2 \mathrm{~h}, 4 \mathrm{~h}$ e $6 \mathrm{~h}$ ) tratadas termicamente e homogeneizadas foram guardadas e enviadas para as análises por Difração de Raios-X, Infravermelho por Transformada de Fourier e Espectroscopia por Energia Dispersiva.

\subsection{Difração de Raios-X}

As análises por DRX foram realizadas no Difratômetro de Raios- X Empyrean PANalytical com tubo de $\mathrm{CuK} \alpha\left(1.5418 \AA\right.$ ) com passo de $0.02 \theta / \mathrm{s}$ e com ângulos de varredura de $10^{\circ} \mathrm{a} 100^{\circ}$.

\subsection{Espectroscopia na região do infravermelho por transformada de Fourier}

As análises por FT-IR foram realizadas em Espectômetro ThermoElectron modelo Nicolet iS10 com 32 varreduras na faixa de 4000 a $400 \mathrm{~cm}^{-1} \mathrm{e}$ resolução de $4 \mathrm{~cm}^{-1}$. Cada amostra foi preparada em pastilha de $\mathrm{KBr}$ na proporção 1:100 de amostra.

\subsection{Espectroscopia por Energia Dispersiva}

As análises foram realizadas seguindo os parâmetros no instrumento Shimadzu Europe EDX-720 à vácuo, collimator $10 \mathrm{~mm}$ e sem spin.

\section{RESULTADOS E DISCUSSÃO}

\subsection{Caracterização térmica}

$\mathrm{O}$ resultado da caracterização térmica é apresentado na figura 1 . A curva, no patamar de $100^{\circ} \mathrm{C}$, apresenta o percentual de $86 \%$ da massa original, aproximadamente, sendo essa perda correspondente a remoção de umidade da amostra [17]. Segundo TORRES (2012), essa perda está associada a desnaturalização do colágeno que constitui a estrutura interna da escama do Arapaima gigas [18] [19]. A respeito da desnaturalização ocorre a separação de hélices triplas em hélices individuais, no qual quando o colágeno é aquecido, a clivagem na ligação de hidrogênio intramolecular induz o encolhimento das fibras de colágeno, havendo em seguida a solubilização e gelatinização (encolhimento da fibra e dispersão em água) [17]. Em torno de $300^{\circ} \mathrm{C}$, observase uma alta taxa de degradação que se segue até $700^{\circ} \mathrm{C}$, no qual é apresentado o valor de $43 \%$ da massa original. Com isso, a curva termogravimétrica estabeleceu o patamar de $700^{\circ} \mathrm{C}$ para o tratamento térmico das escamas, neste caso para estudar a possibilidade do percentual restante de $43 \%$ ser da fase de hidroxiapatita.

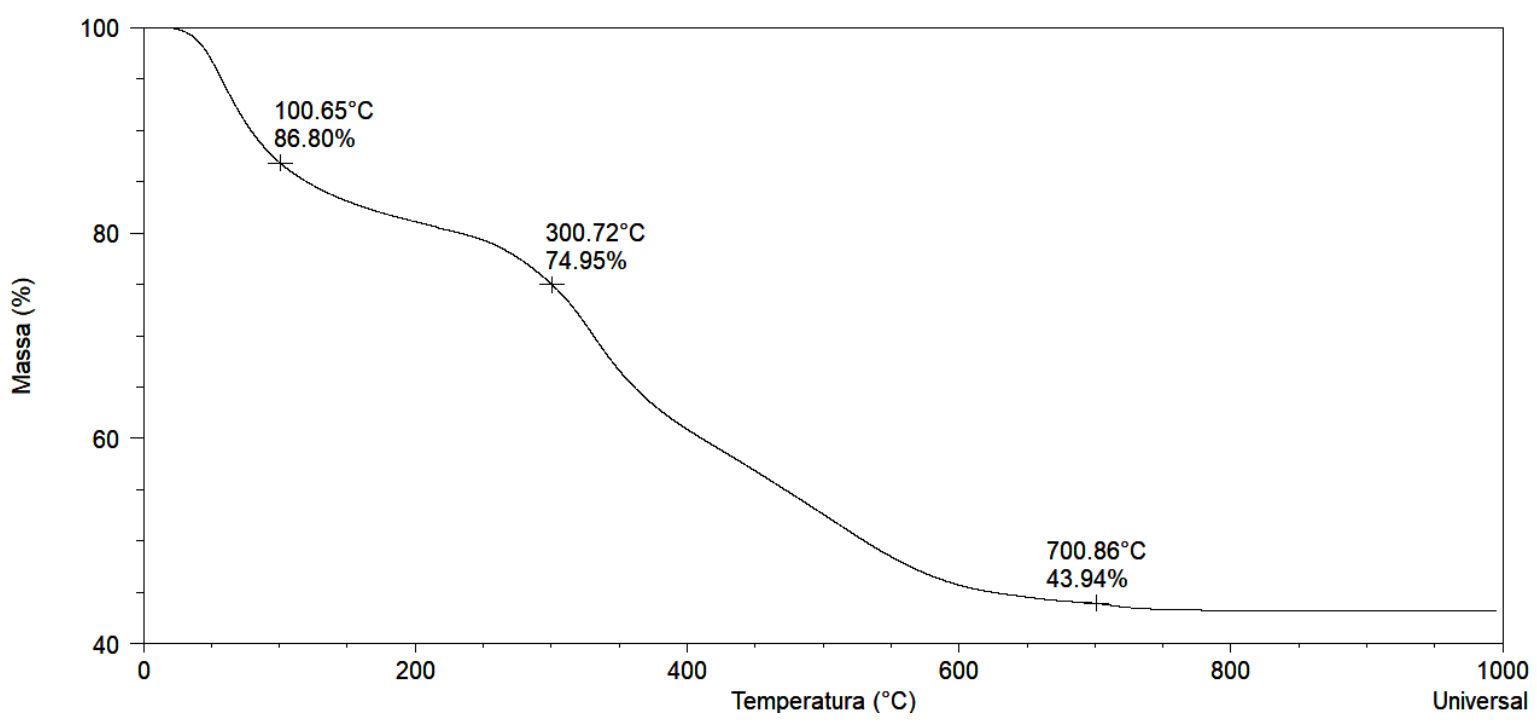


Figura 1: Curva termogravimétrica: massa $(\%)$ em função da temperatura $\left({ }^{\circ} \mathrm{C}\right)$.

\subsection{Difração de raios-x}

Na figura 2 são apresentados os padrões de difração de raios-x das amostras calcinadas à $700^{\circ} \mathrm{C}$ nos tempos de permanência de 2, 4 e 6 horas. Com 2 horas de permanência (curva preta), o material calcinado apresenta um perfil semelhante ao padrão ICSD-26204, sendo este estabelecido como o padrão de DRX da hidroxiapatita. [20] [21] [22]

O tratamento de dados realizado nos padrões indica a presença da fase de HAp com percentual de 99,1\%. A comparação das três curvas apresenta perfis semelhantes em todos as amostras, logo, considerando a economia de tempo, o patamar de 2 horas de permanência é indicado para a obtenção da hidroxiapatita natural por meio do tratamento térmico das escamas. No intervalo de $2 \Theta=10^{\circ}$ e $2 \Theta=60^{\circ}$, todos os planos indicados nas curvas em azul, vermelho e preta são comparáveis com padrão de difração de raios-x da literatura.

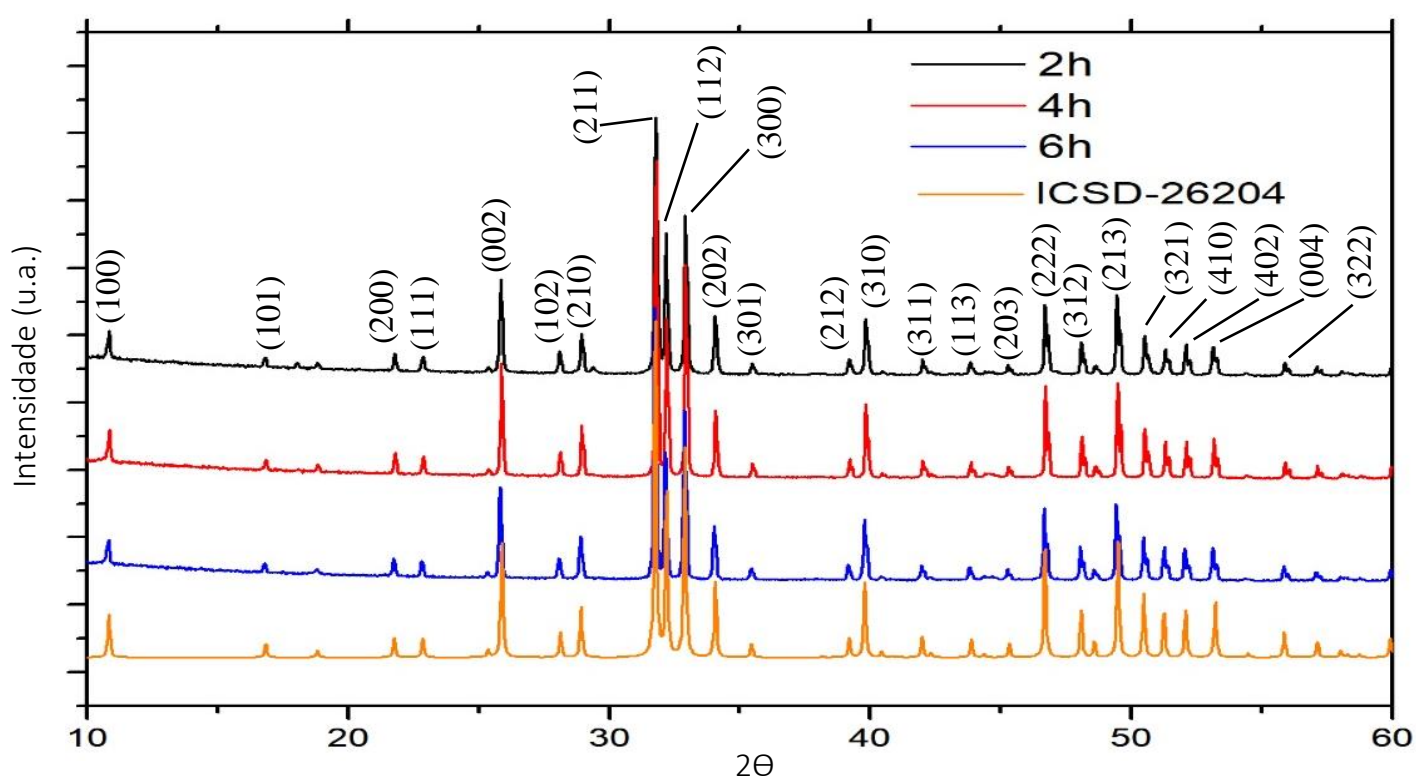

Figura 2: Padrões de DRX das amostras tratadas termicamente por 2, 4 e 6 horas $\left(700^{\circ} \mathrm{C}\right)$ e padrão ICSD:26204. [20] [21] [22]

\subsection{Espectroscopia na região do infravermelho}

A figura 3 apresenta os espectros na região do infravermelho para as amostras 2, 4 e 6 horas $\left(700^{\circ} \mathrm{C}\right)$ para fins de comparação. Verifica-se o mesmo perfil em todas as amostras e são compatíveis com os dados da literatura que apresentam os grupos funcionais presentes na composição química da hidroxiapatita (Tabela 1). [21] [23] [24] 


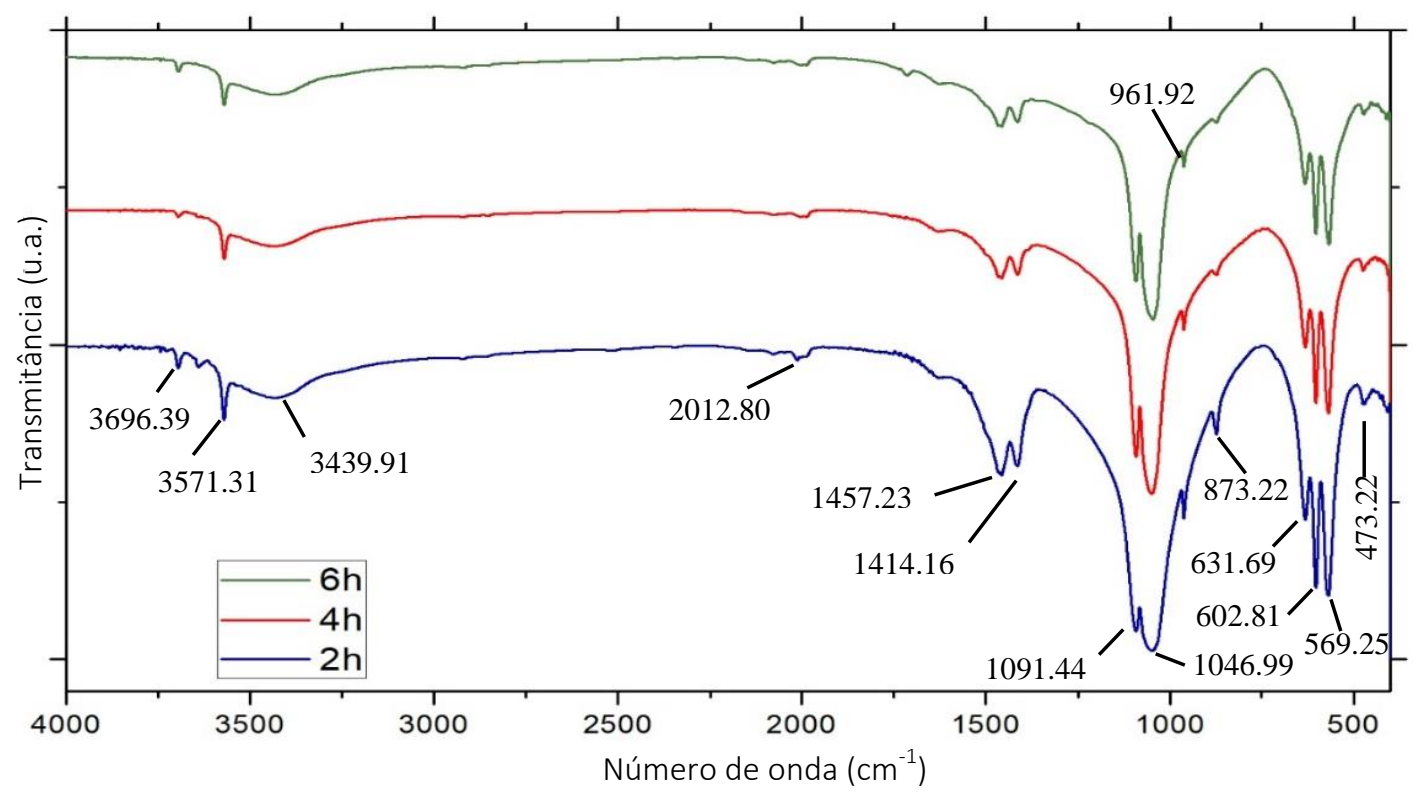

Figura 3: Espectros de transmitância nas amostras de 2, 4 e $6 \mathrm{~h}\left(700^{\circ} \mathrm{C}\right)$.

Tabela 1: Bandas de absorção na região do infravermelho conforme apresentado na figura 3. [24]

\begin{tabular}{c|c}
\hline Número de onda $\left(\mathbf{c m}^{-1}\right)$ & Grupo característico \\
\hline 3577 & Vibração $\mathrm{OH}$ \\
\hline 3447 & Estiramento $-\mathrm{OH}$ \\
\hline 1636 & Deformação H-O-H \\
\hline 1420 & Deformação axial da ligação C=O \\
\hline $1098 / 1043 / 963$ & Estiramento assimétrico P-O \\
\hline 875 & Estiramento Antissimétrico de $\mathrm{CO}_{3}{ }^{-2}$ \\
\hline 630 & Estiramento OH \\
\hline 602 & Deformação O-P-O em $\mathrm{HPO}_{4}{ }^{-2}$ \\
\hline 565 & Deformação angular assimétrica O-P-O \\
\hline
\end{tabular}

Com base na figura 3 e na tabela 1 , os picos no intervalo $3750-3500 \mathrm{~cm}^{-1}$ indicam a presença de íons de $\mathrm{OH}^{-}$, no intervalo $1500-1250 \mathrm{~cm}^{-1}$ e no pico $873 \mathrm{~cm}^{-1}$ caracterizam íons $\mathrm{CO}_{3}{ }^{2-}$ e nos intervalos $1000-900 \mathrm{~cm}^{-1} \mathrm{e}$ $610-560 \mathrm{~cm}^{-1}$ apontam a presença dos íons $\mathrm{PO}_{4}{ }^{3-}$ [23]. Os picos na banda entre $1450-1400 \mathrm{~cm}^{-1}$ são resultado da adsorção de dióxido de carbono presente no ar atmosférico, ocasionando um alargamento da área dos picos devido a sobreposição dos mesmos [20] [21]. Segundo SOUZA (2019), a presença de picos no intercalo de $1420 \mathrm{~cm}^{-1}$ e $1454 \mathrm{~cm}^{-1}$ é referente a impurezas de carbonatos, sendo sugerido que a amostra apresenta apatita moderadamente carbonatada. [24]

\subsection{Espectroscopia por energia dispersiva}

A tabela 2 apresenta a composição elementar das amostradas tratadas termicamente por 2 e 6 horas. Nota-se a presença do elemento de fase secundário de $\mathrm{Na}$ e indicada a concentração de $\mathrm{C}$ nas amostras, o que corrobora com os resultados de espectroscopia na região do infravermelho que supõem, através dos picos da Figura 3, 
que a amostra se trata da hidroxiapatita carbonada seguindo a substituição parcial dos sítios iônicos $\mathrm{PO}_{4}{ }^{3-} \mathrm{e}$ $\mathrm{OH}^{-}$por íons carbonatos, sendo resultados que supõem que a amostra possui mesma composição química presente na apatita que constituem ossos e dente [24]. Além disso, os resultados apresentam altas concentrações de $\mathrm{Ca}, \mathrm{P}$ e $\mathrm{O}$ e não há variação significativa na razão $\mathrm{Ca} / \mathrm{P}$ para as amostras com diferentes tempos de tratamentos térmicos.

Tabela 2: Resultado quantitativo em percentual da composição das amostras de HAp obtidas no MEV/EDS com tratamento térmico alcalino.

\begin{tabular}{c|c|c}
\hline Amostra & $\mathbf{7 0 0} / \mathbf{2 h}(\%)$ & $\mathbf{7 0 0} / \mathbf{6 h}(\%)$ \\
\hline $\mathrm{Ca}$ & 37,27 & 34,53 \\
\hline $\mathrm{P}$ & 13,16 & 12,15 \\
\hline $\mathrm{O}$ & 40,62 & 46,12 \\
\hline $\mathrm{C}$ & 8,29 & 4,86 \\
\hline $\mathrm{Na}$ & 0,67 & 2,33 \\
\hline $\mathrm{Ca} / \mathrm{P}$ & 2,83 & 2,84 \\
\hline
\end{tabular}

\section{CONCLUSÕES}

A utilização das escamas de pirarucu como fonte alternativa para a obtenção de hidroxiapatita natural mostrou-se viável visto que se trata de um procedimento experimental com uso de matéria prima de baixo custo. $\mathrm{O}$ material tratado termicamente e caracterizado estruturalmente e quimicamente apresentou integridade físico-química compatível com a fase cristalina de hidroxiapatita e com baixo teor de impurezas.

Os padrões de difração de raios-x e os espectros na região do infravermelho corroboram em apresentar perfis de curva semelhantes aos encontrados na literatura, assim como os resultados coletados na espectroscopia por energia dispersiva que apresentam as composições elementares compatíveis com a fase HAp, de modo a consolidar a viabilidade de extrair hidroxiapatita natural de escamas de Arapaima gigas.

\section{AGRADECIMENTOS}

Conselho Nacional de Pesquisa e Desenvolvimento Tecnológico (CNPq); Laboratório de Materiais da Amazônia e Compósitos (LaMAC); Laboratório de Materiais, Departamento de Engenharia de Materiais (Universidade Federal do Rio Grande do Norte); Laboratório de Ensaios de Materiais, Faculdade de Tecnologia (Universidade Federal do Amazonas).

\section{BIBLIOGRAFIA}

[1] MORAES, A., BIERHALZ, A., PIRES, A. "Biomateriais: Tipos, aplicações e mercado", Química Nova, v. 38, n. 7, pp. 957-971, 2015.

[2] FAO. Biannual Report on global food markets. Food and Aquaculture Organization of the United Nations. Rome, p. 141, 2016.

[3] WEISS, L. A., MARENGONI, N. G., OLIVERIA, N. T. E., et al., "Resíduos da filetagem industrial de tilápia do Nilo: valores de micro, macronutrientes e aminoácidos e perspectivas de utilização de escamas", Dimensões Tecnológicas e Sociais da Zootecnia, v. 1, pp.1-3, 2015.

[4] FARA, A., ABDULLAH, H. "Characterization of derived natural hydroxyapatite (HAp) obtained from different types of tilapia fish bones and scales", American Institute of Physics, v. 1669, n. 1, pp. 1-6, 2015.

[5] PRASAD, A., DEVENDAR, B., SANKAR, M. R., et al., "Micro-scratch based tribological characterization of hydroxyapatite (HAp) fabricated through fish scales", Materialstoday: Proceedings, 2015.

[6] BRUNDAVANAM, R. K., et al., "Modelling the Crystal Structure of a $30 \mathrm{~nm}$ Sized Particle based Hydroxyapatite Powder Synthesised under the Influence of Ultrasound Irradiation from X-ray powder Diffraction Data”, American Journal of Materials Science, v. 3, n. 4, pp. 84-90, 2013. 
[7] SADAT-SHOJAI, M., KHORASANI, M. T., DINPANAH-KHOSHDARGI, E., et al., "Synthesis methods for nanosized hydroxyapatite with diverse structures", Acta Biomaterialia, v. 9, pp. 7591-7621, 2013.

[8] TANG, M., KRISHNAMRA, N., THONGBUNCHOO, J., et al., "Hydroxyapatite from fish scale for potential use as bone scaffold or regenerative material", Materials Science \& Engineering, v. 62, pp. 183-189, 2015.

[9] ESTEVES, A. D., LOPES, J., OLIVEIRA, J. Sintese e caracterização de pós de hidroxiapatita [cal0(po4)6 (oh)2] obtidas a partir do processo sol-gel. Campinas, FOCO - Ano 6 - n. 8, 2015.

[10] MUHAMMAD, N., GAO, Y., IQBAL, F., et al., Extraction of biocompatible hydroxyapatite from fish scales using novel approach of ionic liquid pretreatment, Elsevier: Separation and Purification Technology, p. 7,2015

[11] SILVA, A. M., DUNCAN, W. L. "Aspectos biológicos, ecologia e fisiologia do pirarucu (Arapaima gigas): uma revisão da literatura", Scientia Amazonia, v. 5, n. 3, pp. 31- 46, 2016.

[12] OLIVEIRA, P., JESUS, R. S., BATISTA, G. M., et al., "Avaliação sensorial, físicoquímica e microbiológica do pirarucu (Arapaima gigas) durante estocagem em gelo", Brazilian Journal of Food Technology, v. 17, 2014.

[13] SILVA, N. D., SILVA, A., BRAGA, T., et al., "Diagnóstico do comércio de Pirarucu nos mercados e feiras de Santarém”, Pará. Biota Amazônia: Open Journal System, v. 6, n. 4, pp. 49-53, 2016.

[14] NUNES, E., FRANCO, R., MÁRSICO, E. "Qualidade do pirarucu (Arapaima gigas) salgado seco comercializado em mercados varejistas", Ver. Inst. Adolfo Lutz, v. 71, n. 3, pp. 520-529, 2012.

[15] KRISTEN, I., RUCK, L., MATEUS, L., et al., "A pesca do pirarucu (Arapaima sp.) na bacia do rio Araguaia em Mato Grosso-Brasil”, Boletim do Instituto de Pesca, v. 38, n. 2, pp. 131-144, 2012.

[16] CASTELLO, L., ARANTES, C., MCGRATH, D., et al., "Understanding fishing-induced extinctions in the Amazon"., Aquatic Conserv: Mar. Freshw. Ecosyst, v.25, n.5, 2014.

[17] TORRES, F., TRONCOSO, O., AMAYA, E. (s.d.). "The effect of water on the thermal transitions of fish scales from Arapaima gigas", Elsevier: Materials Science and Engineering C, v. 32, n. 8, pp. 2212-2214, 2012.

[18] MEYERS, M.A., LIN, Y.S., OLEVSKY, E.A., et al., "Battle in the Amazon: arapaima versus Piranha", Adv. Eng. Mater., v.14, pp. 279-288, 2012.

[19] YANG, W., CHEN, I.H., GLUDOVATZ, B., et al., "Natural flexible dermal armor", Adv. Mater., v. 25, pp. 31-48, 2013.

[20] GAO, X., DAI, C., LIU, W., et al., "High-scale yield of nano hydroxyapatite through combination of mechanical activation and chemical dispersion", Biomaterials Synthesis and Characterization, v.28, n.6, 2017.

[21] MONDAL, B., MONDAL, S., MONDAL, A., et al., "Fish scale derived hydroxyapatite scaffold for boné tissue engineering", Elsevier: materials characterization, v. 121, pp. 112-124, 2016.

[22] KONGSRI, S., JANPRADIT, K., BUAPA, K., et al., "Nanocrystalline hydroxyapatite from fish scale waste: Preparation, characterization and application for selenium adsorption in aqueous solution", Elsevier: Chemical Engineering Journal, v. 215-216, pp. 522-532, 2013.

[23] PANDA, N.N., PRAMANIK, K., SUKLA, L.B. "Extraction and characterization of biocompatible hydroxyapatite from fresh water fish scales for tissue engineering scaffold", Bioprocess and Biosystems Engineering, v. 37, n. 3, pp. 433-440, 2013.

[24] SOUZA, T.S., et al . "Síntese, caracterização e modificação da hidroxiapatita com zinco para aplicação na reação de esterificação", Matéria (Rio J.), Rio de Janeiro, v. 24, n. 1, e-12312, 2019.

\section{ORCID}

Mateus Oliveira de Amorim Karyane Meazza

Ana Alice Oliveira

José Carlos Calado Sales Júnior https://orcid.org/0000-0002-6254-2222

https://orcid.org/0000-0001-6729-1453

https://orcid.org/0000-0001-9826-3172

https://orcid.org/0000-0001-9785-0447 
Yurimiler Leyet Ruiz

Jean Carlos Silva Andrade https://orcid.org/0000-0002-5774-5391

https://orcid.org/0000-0002-6777-4276 